\title{
RETRIEVAL TECHNIQUE FOR THE DIAGNOSIS OF SOLID BREAST TUMORS ON SONOGRAM
}

\author{
Wen-Jia Kuo, ${ }^{*}$ Ruey-Feng Chang, ${ }^{*}$ Cheng Chun Lee, ${ }^{\dagger}$ Woo Kyung Moon ${ }^{\S}$ and \\ DAR-REN CHEN ${ }^{*}$ \\ *Department of Computer Science and Information Engineering, National Chung Cheng University, Chiayi, Taiwan; \\ Departments of ${ }^{\dagger}$ Medical Research and ${ }^{\ddagger}$ General Surgery, China Medical College and Hospital, Taichung, Taiwan; \\ and ${ }^{\S}$ Department of Diagnostic Radiology, College of Medicine, Seoul National University Hospital, \\ Seoul, South Korea
}

(Received 12 October 2001; in final form 23 April 2002)

\begin{abstract}
We evaluated a series of pathologically proven breast tumors using an image-retrieval technique for classifying benign and malignant lesions. A total of 263 breast tumors (129 malignant and 134 benign) were retrospectively evaluated. The physician located regions-of-interest (ROI) of ultrasonic images and texture parameters (contrast, covariance and dissimilarity) were used in the process of the content-based image-retrieval technique. The accuracy of using the retrieval technique for classifying malignancies was $92.55 \%(236$ of 255$)$, the sensitivity was $94.44 \%$ (119 of 126), the specificity was $90.70 \%$ (117 of 129), the positive predictive value was 90.84\% (119 of 131), and negative predictive value was $94.35 \%$ (117 of 124) for the proposed computer-aided diagnostic system. This computer-aided diagnosis system can provide a useful tool and its high negative predictive value could potentially help avert benign biopsies. It is unnecessary to perform any training procedures. This computer-aided diagnosis system can provide a second opinion for a sonographic interpreter; the main advantage in this proposed system is that we do not need any training. Historical cases can be directly added into the database and training of the diagnosis system again is not needed. With the growth of the database, more and more information can be collected and used as reference cases while performing diagnoses. This increases the flexibility of our diagnostic system. (E-mail: dlchen88@ms13.hinet.net) (c) 2002 World Federation for Ultrasound in Medicine \& Biology.
\end{abstract}

Key Words: Image retrieval, Sonogram, Computer-aided diagnosis.

\section{INTRODUCTION}

The most useful way to decrease deaths from breast cancer is to treat the disease as early as possible. However, earlier treatment requires early diagnosis, and early diagnosis requires an accurate and reliable diagnostic procedure. Currently, the most frequently- adopted imaging studies for early detection and diagnosis of breast cancers include mammography and sonography. Breast ultrasound (US) is used as an adjunct to mammography, to help to distinguish benign from malignant lesions. Mammography gets all the publicity, whereas breast US does its job quietly behind the scenes (Raymond 2000). Recent progress in computer science and technical advances in US have expanded the potential usefulness of this modality for the evaluation of breast lesions. The

Address correspondence to: Dar-Ren Chen, M.D., Department of General Surgery, China Medical College \& Hospital, 2 Yer-Der Rd. E-mail: dlchen88@ms13.hinet.net role of diagnostic breast US is no longer to be restricted to the differentiation between cysts and solid tumors. Using the current real-time US is more convenient than mammography in the evaluation of a breast mass in daily clinical practice. However, breast cancer is so heterogeneous that there is considerable overlap in the sonographic characteristics between malignant and benign lesions. This is the main reason for the controversial area in its utility for classifying breast tumors. A study by Stavros et al. (1995) had expanded the role of breast US. However, the sonographic technique described by these authors required extensive real-time evaluation by an experienced interpreter; this may not be practical for most clinical settings. In most hospitals, mammography and sonography are supplied for doctors to diagnose via visual experiences to increase the diagnostic confidence and decrease benign biopsies. Compared with other procedures, biopsy is expensive because of the large number of indeterminate lesions that need to be differentiated per 
year. Therefore, it is advantageous to diminish the use of invasive methods of distinguishing malignant from benign masses in the breast, to reduce the number of unnecessary biopsies, allay anxiety and control costs. Moreover, most biopsies might be avoidable because the rate of positive findings at biopsy for cancer is low, between 10\% and 31\% (Bassett et al. 1991; Gisvold and Martin 1984; Rosenberg et al. 1987). Contrast-enhanced magnetic resonance imaging (MRI) (Gilles et al. 1994; Stomper et al. 1995), and breast scintigraphy (Palmedo et al. 1996; Stomper et al. 1995; Tiling et al. 1997) have been discussed in recent reports as possible alternatives to excision biopsy for those benign and probably benign lesions. However, these modalities are considered as second-line modalities.

In recent years, the number of digital images has enormously increased for many applications. As image databases become larger and more widespread, there is a growing demand for effective and flexible techniques to retrieve images automatically from the database. This image retrieval needs to find from the database images that are the same as (or images with high similarity to) the query image provided by users. Thus, images that satisfy a perceptual similarity with the user's query could be retrieved from the database. The process of contentbased image retrieval has been investigated in several recent efforts. Currently, finding effective methods to incorporate content-based image retrieval approaches into an image database system is attracting research efforts (Gudivada and Raghavan 1995; Hafner et al. 1995; Ogle and Stonebraker 1995; Srihari 1995; Gimel'farb and Jain 1996). In this study, we evaluated a series of pathologically proven breast tumors using an image-retrieval technique for classifying benign and malignant lesions.

\section{MATERIALS AND METHODS}

In this work, a physician first extracted the subimage of the region-of-interest (ROI), and then the computer analyzed the subimage, using intensity variation and texture information.

The ultrasonic image database included 263 sonographic images of pathologically proven benign breast tumors from 134 patients, and carcinomas from 129 patients (tumor size $>0.8 \mathrm{~cm}$ in all cases). The database contained only one image from each patient. The ultrasonic images were captured at the largest diameter of the tumor. The images were collected from August 1, 1999 to May 31, 2000; the patients' ages ranged from 18 to 64 years. Sonography was performed with a high-resolution near-field HDI 3000 system and a L10-5 $38 \mathrm{~mm}$ linear transducer with freeze-frame capability (ATL, Bothel, WA). Dynamic range was set at $55 \mathrm{~dB}$. No acoustic

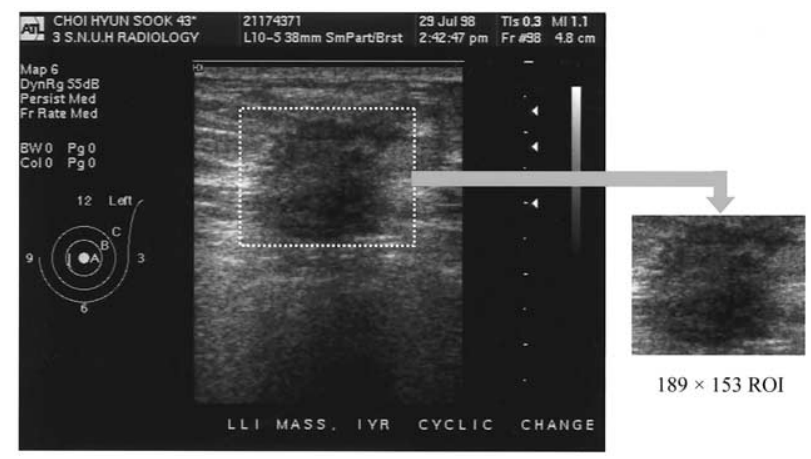

Fig. 1. A $640 \times 480$ digital image captured from the US scanner. In a $1 \mathrm{~cm} \times 1 \mathrm{~cm}$ rectangle, there are $94 \times 94=8836$ pixels. The ROI rectangle is $2.01 \mathrm{~cm} \times 1.63 \mathrm{~cm}$ and $189 \times 153$ pixels.

stand-off pad was used in any of the cases. The sonographic gain setting remained unchanged throughout the entire period of study, except for changes made to obtain the best view. The entire database was supplied by one of the coauthors, Dr. Moon from the Department of Diagnostic Radiology, Seoul National University Hospital, Seoul, South Korea. All solid nodules at US belong over C3 according to ACR BI-RADS category, and all images were fed into the algorithm without selection bias. Most of the cases were in the category of indeterminate or malignant because we included only histologically confirmed cases.

The monochrome ultrasonic image was quantized into 8 bits (i.e., 256 grey levels) and the features were stored via the magneto-optical disks. The entire database was supplied from other (country) hospitals, and the ROI was also manually selected using the ProImage package also by the breast surgeon, who was familiar with mammosonographic interpretations but unfamiliar with the tissue diagnosis and cell type before ROI selection. The ROI subimage was then saved as a file for later analysis. ROI images were used in our breast image database to further to investigate the texture characteristics of benign and malignant tumors. Figure 1 shows an example of a $640 \times 480$ digital image captured from the US scanner. In a $1-\mathrm{cm} \times 1-\mathrm{cm}$ rectangle, there were $94 \times 94=8836$ pixels. The ROI rectangle was $1.65 \mathrm{~cm} \times 0.96 \mathrm{~cm}$ and $155 \times 90$ pixels.

For the purpose of representing properties of US texture, many researches about US texture analysis have been proposed over the last 20 years (McPherson et al. 1986; Goldberg et al. 1992; Garra et al. 1993; Petrick et al. 1996; Vittitoe et al. 1997). For texture analysis, the most useful features were those directed from co-occurrence matrices of the image described as follows.

A co-occurrence matrix, $P_{\phi, d}(i, j)$, is a matrix in which the $(i, j)$ th element describes the frequency of 
occurrence of two pixels that are separated by distance $d$ in the direction $\phi$ with grey levels $i$ and $j$. For an $N \times N$ region with $M$ grey levels $(0, \ldots, M-1)$, the grey-level differences that single pairs of pixels can exhibit are:

$$
\begin{array}{cccc}
(0,0) & (0,1) & \cdots & (0, M-1) \\
(1,0) & (1,1) & \cdots & (1, M-1) \\
\vdots & \vdots & \vdots & \vdots \\
(M-1,0) & (M-1,1) & \cdots & (M-1, M-1)
\end{array}
$$

We can find that the texture variations in a region can be captured through the co-occurrence matrix by various $\phi$ and $d$. That is, the co-occurrence matrix characterizes the spatial interrelationships of the grey levels in a texture pattern and it is invariant under monotonic grey-level transformations. In general, the minimum amount required for the set of co-occurrence matrices is four $\left(\phi=0^{\circ}, 45^{\circ}, 90^{\circ}\right.$ and $135^{\circ}$, respectively; $\left.d=1\right)$ for the texture, about which we have no prior knowledge. However, it is obvious that the size of the matrix is a function of the number of grey levels in the image and it would be prohibitively expensive to evaluate a matrix for each pixel in a general 8 -bit image $(256 \times 256$ elements in a co-occurrence matrix). Thus, we use the statistical parameter matrices (Roux and Coatrieux 1997) with the same texture reservation properties, instead of the cooccurrence matrix.

Contrast, covariance and dissimilarity are the statistical methods we adopted that can evaluate the texture parameters for several distances between pixels and directly from the image, without using co-occurrence matrices. The main advantage of these methods resides in the calculation cost, which depends only on the size of the image treated and not on the number of grey levels. Moreover, visually perceptible physical parameters can be extracted from the image, such as contrast, granularity, regularity, periodicity, finesse or coarseness of the texture, and so on. We briefly introduce the evaluation of texture parameters as follows.

Let $S$ be a region of an image, $g(i, j)$ be the intensity value at the position $(i, j)$ of $S, \delta=(\Delta i, \Delta j)$ be the distance between two pixels and $\eta$ be the grey-level average of the region $S$. For a given value of $\delta$, contrast, covariance and dissimilarity are defined as follows.

Contrast

$$
\operatorname{Con}(\delta)=E\{g(i, j) \cdot g(i+\Delta i, j+\Delta j)\}
$$

\section{Covariance}

$$
\operatorname{Cov}(\delta)=E\{[g(i, j)-\eta] \cdot[g(i+\Delta i, j+\Delta j)-\eta]\}
$$

Dissimilarity

$$
\operatorname{Diss}(\delta)=E\{g(i, j)-g(i+\Delta i, j+\Delta j)\}
$$

According to our experimental results, we find that the covariance performs better than other texture parameters. In this study, these three texture parameters, contrast, covariance and dissimilarity, were used as the features and were evaluated with different weighting coefficients for further diagnosis. In this work, a sonographic image produced $5 \times 5$ texture parameters with $\Delta i$ and $\Delta j=1$, $2, \ldots, 5$.

The distance measure that we used to retrieve images from the database for diagnosis is defined as follows:

$$
\operatorname{dis}=\sum_{i=1}^{3} w_{i}\left(Q_{i}-f_{i}\right)^{2}
$$

Where $A_{i}$ are the feature values of the query image (the image to be diagnosed) and $f_{i}$ are the feature values of the image in the database. The feature values are the three texture parameters, contrast, covariance and dissimilarity; each contains 24 coefficients. Weighting coefficients $w_{i}$ for $i=1,2,3$ are used with different values between 0 and 1 , and the summation of weighting coefficients is equal to 1 . The weighting coefficient for covariance parameters is set to a larger number because the covariance performs better than the other two features.

In this study, we retrieved the first $K$ candidates with smaller distance values from the image database for differential diagnosis. The tumor was then differentiated as benign or malignant according to the $D S$ value, by evaluating the first $K$ candidates in sequence, and the $D S$ value was defined as follows.

$$
D S=\sum_{i=1}^{K} \frac{K-i+1}{\operatorname{Sum}_{K}} \times \text { Tumor_class, }
$$

and

Tumor_class $=\left\{\begin{array}{l}1, \text { if the retrieved result is malignant } \\ 0, \text { if the retrieved result is benign }\end{array}\right.$,

where $\operatorname{Sum}_{k}$ is the summation of 1 to $K$. If the evaluated $D S$ value is larger than a threshold $T H$, the tumor is classified as malignant. Conversely, if the evaluated $D S$ value is less than a threshold $T H$, the tumor is classified as benign. 
Traditionally, the tumor is classified as benign or malignant in many computer-aided diagnostic (CAD) systems (Chen et al. 1999, 2000a, 2000b). In fact, there exist some tumors residing within the boundary condition that should be further differentiated by the doctor. To get more interactive applications of the proposed system, it is worthwhile to reserve the tumors within the boundary condition as indeterminate class as we used in daily clinical practice, which indicate warranting biopsy. Thus, the threshold $T H$ is modified as $T H_{m}$ and $T H_{b}$. If the evaluated $D S$ value is larger than a threshold $T H_{m}$, the tumor is classified as malignant. Conversely, if the evaluated $D S$ value is less than a threshold $T H_{b}$, the tumor is classified as benign. Those cases that do not belong to benign or malignant are then classified as indeterminate.

Generally speaking, the histogram distribution of an image usually has similar properties when they belong to the same family. In the ultrasonic images, most of the benign or malignant cases have a similar distribution with the same family. Thus, according to the following definition, we can modify the distribution into similar mean and variance. The definition is as follows.

Let $f(x)$ be any pdf. Then, for any $\mu,-\infty<\mu<\infty$, and any $\sigma>0$, the family of pdfs $(1 / \sigma) f((x-\mu) / \sigma)$, indexed by the parameter $(\mu, \sigma)$, is called the locationscale family with standard pdf $f(x) ; \mu$ is called the location parameter and $\sigma$ is called the scale parameter.

To reduce the factors when different physicians operate the ultrasonic system, such as contrast, brightness and so on, we used the above properties to adjust the ultrasonic images into the family with almost the same mean and variance in this study.

Quantization is the process of representing a set of continuous-valued samples by a finite number of states. If each sample is quantized independently, then the process is known as scalar quantization. A scalar quantizer $Z(\cdot)$ is a function that is defined in terms of a finite set of decision levels $d_{i}$ and reconstruction levels $r_{i}$, given by:

$$
Q(s)=r_{i} \text {, if } s \in\left(d_{i-1}, d_{i}\right], i=1, \ldots, L
$$

where $L$ is the number of output states. That is, the output of the quantizer is the reconstruction level $r_{i}$, if $s$, the value of the sample, is within the range $\left(d_{i-1}, d_{i}\right)$.

In the texture parameters, the values were directly evaluated by the grey level of the pixel. However, little difference in grey level will still make a large difference for the texture parameters. Thus, we use quantization to reduce this situation, for the reason that the parameter in which we are interested is their comparative relationship. The step size of quantization is decided by equal cumulative number of the pixels into $K$ intervals. That is, each interval will have the same number of accumulations.
Table 1. The performance for different texture parameters, contrast, covariance and dissimilarity

\begin{tabular}{lccc}
\hline \multicolumn{1}{c}{ Item } & Contrast & Covariance & Dissimilarity \\
\hline Accuracy (\%) & 86.94 & 88.89 & 54.86 \\
TN & 115 & 112 & 48 \\
FP & 26 & 21 & 43 \\
TP & 118 & 120 & 48 \\
FN & 9 & 8 & 36 \\
\hline
\end{tabular}

The simulations were made on a single CPU Intel Pentium-II ${ }^{\circledR} 400-\mathrm{MHz}$ personal computer with Microsoft Windows $98^{\circledR}$ operating system.

\section{RESULTS}

Accuracy, sensitivity, specificity, positive predictive value and negative predictive value are the five most generally used objective indices to estimate the performance of diagnosis results. Table 1 shows the performance of three texture parameters. We can find that the covariance and contrast performed better than the feature of dissimilarity. Table 2 shows the comparison of different sets of weighting coefficients for the three texture parameters. A different sets of coefficients had a different diagnostic performance. Although the results of all these listed five sets of coefficients were acceptable, we found that the ideal set of coefficients were 0.3 (contrast), 0.7 (covariance), and 0 (dissimilarity), because of their good performance in average and lower indeterminate case numbers. Table 3 lists the percentile of Table 2. Table 4 lists the case numbers of false-negative results (i.e., the number of cancers that were misdiagnosed as benign) in each different threshold value of benignancy. Regarding the diagnostic performance of false-negative, true-negative, indeterminate, sensitivity and specificity, the benign threshold cut-off value of 0.45 was the choice. Table 5 summarises the diagnostic performance of using selected weighting coefficients $(0.3,0.7,0)$ and cut-off values of threshold $(<0.45$ for benign and $>0.55$ for

Table 2. Diagnostic performance using different sets of weighting coefficients of the three texture parameters (contrast, covariance and dissimilarity) for the proposed retrieval technique

\begin{tabular}{lrrrrr}
\hline $\begin{array}{c}\text { Weighting } \\
\text { coefficients }\end{array}$ & TN & FP & FN & TP & $\begin{array}{c}\text { Indeterminate } \\
\text { (benign vs. } \\
\text { malignant) }\end{array}$ \\
\hline $0.1,0.9,0$ & 112 & 14 & 5 & 118 & $14(8,6)$ \\
$0.2,0.8,0$ & 115 & 13 & 7 & 119 & $9(6,3)$ \\
$0.3,0.7,0$ & 117 & 12 & 7 & 119 & $8(5,3)$ \\
$0.4,0.6,0$ & 119 & 9 & 8 & 118 & $9(6,3)$ \\
$0.5,0.5,0$ & 119 & 9 & 9 & 116 & $10(6,4)$ \\
\hline
\end{tabular}


Table 3. Diagnostic performance using different sets of weighting coefficients of the three texture parameters (contrast, covariance and dissimilarity) for the proposed retrieval technique

\begin{tabular}{|c|c|c|c|c|c|c|}
\hline Weighting coefficients & $\begin{array}{c}\text { Accuracy } \\
(\%)\end{array}$ & $\begin{array}{c}\text { Sensitivity } \\
(\%)\end{array}$ & $\begin{array}{c}\text { Specificity } \\
(\%)\end{array}$ & $\begin{array}{l}\text { Positive } \\
\text { predictive } \\
\text { value }(\%)\end{array}$ & $\begin{array}{l}\text { Negative } \\
\text { predictive } \\
\text { value }(\%)\end{array}$ & Indeterminate \\
\hline $0.1,0.9,0$ & 92.37 & 95.94 & 88.89 & 89.39 & 95.73 & 14 \\
\hline $0.2,0.8,0$ & 92.13 & 94.44 & 89.84 & 90.15 & 94.26 & 9 \\
\hline $0.3,0.7,0$ & 92.55 & 94.44 & 90.70 & 90.84 & 94.35 & 8 \\
\hline $0.4,0.6,0$ & 93.31 & 93.65 & 92.97 & 92.91 & 93.70 & 9 \\
\hline $0.5,0.5,0$ & 92.89 & 92.80 & 92.97 & 92.80 & 92.97 & 10 \\
\hline
\end{tabular}

malignant). Table 6 gives the percentile of Table 5; all the diagnostic performances were over $90 \%$. In summary, the accuracy of using the retrieval technique for classifying malignancies was $92.55 \%$ (236 of 255 ), the sensitivity was $94.44 \%$ (119 of 126), the specificity was $90.70 \%$ (117 of 129), the positive predictive value was $90.84 \%$ (119 of 131), and negative predictive value was 94.35\% (117 of 124) for the proposed CADx system. The ideal weighting coefficients were $0.3,0.7,0$ for contrast, covariance and dissimilarity, respectively. The threshold values for malignant and benign were 0.55 and 0.45 ; the threshold value in between indicated indeterminate. To increase the diagnostic performance of the CAD system, the group of indeterminates was set aside and excluded in calculating the diagnosis performance. The average execution time for the diagnosis of a case was $0.0025 \mathrm{~s}$. With a sensitivity of $94.44 \%$ and a specificity of $90.70 \%$, our proposed method provides objective evidences for good diagnoses of breast tumors.

\section{DISCUSSION}

Visual features were used in the content-based visual query (CBVQ) techniques to index images and videos for providing search capabilities in a system. The process of content-based image retrieval has been investigated in several recent efforts, such as Virage's commercial image-retrieval system, the Chabot image-retrieval system, IBM's QBIC project, and MIT's Photobook system. The QBIC system has investigated many features such as color, texture, shape, and so on. In addition, the system has placed a research focus on designing strategies for indexing the features. The Photobook system has focused on texture, shape and facial features. In addition, they also investigated new methods for representing image features, not only to provide the discrimination of images, but also to preserve their semantic information. Some systems allowing the user to sketch the outlines of objects to find the desired images are also considered. For example, the QVE (Query by Visual Example) system first normalizes the images in the database as regular-sized images and then processes them to get abstract images. The abstract images are used as the pictorial index. Users sketch rough outlines of objects for the desired image and the rough drawing is matched with the abstract images to find the most similar image. Currently, finding effective methods to incorporate content-based image-retrieval approaches into an image database system is an attractive research.

In recent years, the amount of digital images has enormously increased both in the computer storage and network transmission. As the image databases become larger and more widespread, there is a growing demand for effective and flexible techniques to retrieve images automatically from the database for many applications. Fingerprint matching is one of the good examples. The main goal of image retrieval is to find in the database the same image or the image with the highest similarity to the query image provided by users. Thus, images satisfying the perceptual similarity with the users' query could be retrieved from the database. The most important factor for image retrieval is the retrieval accuracy. However, there is considerable overlap in the sonographic

Table 4. The performance of the 129 malignant cases with different threshold values of benign $\mathrm{TH}_{\mathrm{b}}$

\begin{tabular}{lccccc}
\hline Threshold & $\begin{array}{c}\text { False- } \\
\text { negatives }\end{array}$ & $\begin{array}{c}\text { True- } \\
\text { negatives }\end{array}$ & Indeterminate & Sensitivity (\%) & $\begin{array}{c}\text { Specificity } \\
(\%)\end{array}$ \\
\hline 0.50 & 9 & 118 & 1 & 92.97 & 90.77 \\
0.45 & 7 & 117 & 3 & 94.44 & 90.70 \\
0.35 & 6 & 109 & 4 & 95.20 & 90.08 \\
0.25 & 6 & 105 & 4 & 95.20 & 89.74 \\
0.15 & 4 & 98 & 6 & 96.75 & 89.09 \\
\hline
\end{tabular}


Table 5. Classification of breast nodules by proposed imageretrieval technique with weighting coefficients $0.3,0.7,0$ for contrast, covariance and dissimilarity, respectively

\begin{tabular}{lcc}
\hline & \multicolumn{2}{c}{$\begin{array}{c}\text { Proposed image-retrieval } \\
\text { diagnosis method }\end{array}$} \\
\cline { 2 - 3 } \multicolumn{1}{c}{$\begin{array}{c}\text { Sonographic } \\
\text { classification }\end{array}$} & Benign & Malignant \\
\hline $\begin{array}{l}\text { Benign (DS }<0.45) \\
\text { Indeterminate }\end{array}$ & TN 117 & FN 7 \\
Malignant (DS $\geq 0.55)$ & 5 & 3 \\
Total & FP 12 & TP 119 \\
\hline
\end{tabular}

$\mathrm{TP}=$ true-positive $; \mathrm{TN}=$ true-negative $; \mathrm{FP}=$ false-positive $; \mathrm{FN}=$ false-negative.

characteristics between malignant and benign lesions. It is difficult to classify malignant from benign tumors completely from sonography, either by the naked eye or by computer. Although the radiologist does the diagnostic task with a level of intelligence and flexibility that is difficult to duplicate with a computer, the radiologist's approach is not devoid of limitations. There exist perceptual errors and interobserver variations in the interpretations of sonograms (Blickstein et al. 1995). The computer can be the ally if the complex processes in image perception are deconstructed (Huber et al. 2000).

Texture analysis (i.e., textural information extracted from the image) is one of the of the possible ways to resolve this problem (Shankar et al. 1993; Raeth et al. 1986). However, the selection of textural features will affect the performance of CAD scheme. Texture analysis can be briefly classified into three main groups: there are the models, the mathematical morphology and the statistical method. Markovian model and fractal model are the most frequently discussed models in texture analysis. Mathematical morphology methods are based on the study of shape representation in the image, yet these shapes are not always directly visible. In mathematical morphology, grey-level morphology of images and morphological granulometry are the most two most fre-

Table 6. Performance of the proposed diagnostic system using the image-retrieval technique

\begin{tabular}{lc}
\hline \multicolumn{1}{c}{ Item } & $\begin{array}{c}\text { Proposed image-retrieval } \\
\text { diagnosis method }\end{array}$ \\
\hline Accuracy (\%) & 92.55 \\
Sensitivity (\%) & 94.44 \\
Specificity (\%) & 90.70 \\
Positive predictive value (\%) & 90.84 \\
Negative predictive value (\%) & 94.35 \\
\hline
\end{tabular}

Accuracy $=(\mathrm{TP}+\mathrm{TN}) /(\mathrm{TP}+\mathrm{TN}+\mathrm{FP}+\mathrm{FN})$; sensitivity $=$ $\mathrm{TP} /(\mathrm{TP}+\mathrm{FN})$; specificity $=\mathrm{TN} /(\mathrm{TN}+\mathrm{FP})$; positive predictive value $=\mathrm{TP} /(\mathrm{TP}+\mathrm{FP})$; and negative predictive value $=\mathrm{TN} /(\mathrm{TN}+\mathrm{FN})$. quently adopted methods. For the reason that the natural textures usually have a highly stochastic characterization, whether they are structured or not, it would seem advisable to use statistical measurements to characterise those signals that are insufficiently described by other approaches. In most cases, statistical parameters can be evaluated either at order one, at order two or at higher orders.

Many lesions found by breast US are not clinically apparent by palpation and, in the case of indeterminate lesions, this leaves the physician without diagnostic confidence and the patient with a high degree of anxiety. Improved imaging techniques have allowed the management of sonographically-detected breast lesions to become less invasive. In this study, we propose a novel diagnosis system in which interpixel correlations on the US images were used to differentiate benign and malignant tumors. Three texture features, including contrast, covariance and dissimilarity, and an image-retrieval technique were adopted to achieve better results. Diagnosistic results are not only classified traditionally, into benign or malignant cases. An indeterminate case is added for further differentiation by physicians, to avoid misdiagnosis of the boundary cases. However, the number of indeterminate cases is as few as possible. The main difference in evaluating results in among this study and others is that we do not need any training procedures. With the growth of the database, more and more information can be collected and used as reference cases while performing diagnosis.

\section{REFERENCES}

Bassett LW, Liu TH, Giuliano AI, Gold RH. The prevalence of carcinoma in palpable vs. impalpable, mammographically detected lesions (comment). AJR 1991;157:21-24.

Blickstein I, Goldschmit R, Strano D, et al. Echogenicity of fibroadenoma and carcinoma of the breast: Quantitative comparison using gain-assisted densitometric evaluation of sonograms. J Ultrasound Med 1995;14:661.

Chen DR, Chang RF, Huang YL. Computer-aided diagnosis applied to US of solid breast nodules by using neural networks. Radiology 1999;213:407-412.

Chen DR, Chang RF, Huang YL, et al. Texture analysis of breast tumors on sonograms. Semin Ultrasound CT MR 2000a;4(21):308316.

Chen DR, Chang RF, Huang YL. Breast cancer diagnosis using selforganizing map for sonography. Ultrasound Med Biol 2000b;3: 405-411.

Garra BS, Krasner BH, Horii SC, Ascher S, Mun SK, Zeman RK. Improving the distinction between benign and malignant breast lesions: The value of sonographic texture analysis. Ultrason Imaging 1993; 15:267-285.

Gilles R, Guinebretiere JM, Lucidarme O, et al. Nonpalpable breast tumors: Diagnosis with contrast-enhanced subtraction dynamic MR imaging. Radiology 1994;191:625-631.

Gimel'farb GL, Jain AK. On retrieving textured images from an image database. Pattern Recognit 1996;29:1461-1483.

Gisvold JJ, Martin JJ. Prebiopsy localization of nonpalpable breast lesions. AJR 1984;143:477-481. 
Goldberg V, Maduca A, Ewert DL, Gisvold JJ, Gerrnleaf JF. Improvement specificity of ultrasonography for diagnosis of breast tumors by means of artificial intelliengence. Med Phys 1992;19:14751481.

Gudivada VN, Raghavan VV. Design and evaluation of algorithms for image retrieval by spatial similarity. ACM Trans Inform Syst 1995;13:115-144.

Hafner J, Sawhney HS, Equitz W, Flickner M, Niblack W. Efficient color histogram indexing for quadratic form distance functions. IEEE Trans Pattern Anal Machine Intell 1995;17:729-736.

Huber S, Danes J, Zuna I, Teubner J, Delortue S. Relevance of sonographic B-mode criteria and computer-aided ultrasonic tissue characterization in differential diagnosis of solid breast masses. Ultrasound Med Biol 2000;26(8):1243-1252.

McPherson DD, Aylward PE, Knosp BM, et al. Ultrasound characterization of acute myocardial ischemia by quantitative texture analysis. Ultrason Imaging 1986;8:227-240.

Ogle VE, Stonebraker M. Chabot: Retrieval from a relational database of images. IEEE Comput 1995;28:40-48.

Palmedo H, Grunwald F, Bender H, et al. Scintimammography with technetium-99m methoxyisobutylisonitrile: Comparison with mammography and magnetic resonance imaging. Eur J Nucl Med 1996; 23:940-946.

Petrick N, Chan HP, Wei D, Sahiner B, Helvie MA, Adler DD. Automated detection of breast masses on mammograms using adaptive contrast enhancement and texture classification. Med Phys 1996;23:1685-1696.

Raeth U, Zuna I, Schlaps D, et al. Image texture of normal and pathological ultrasound B-scan-role of histomorphological tissue structure. In: Thijssen J, ed. Proceedings of the Fifth European Communities Workshop. Nijmegen, The Netherlands: University of Njimegen Press, 1986:153.

Raymond HW. Letter from the editor. Semin Ultrasound CT MR 2000;21(4):285.

Rosenberg L, Schwartz GF, Feig SA, Patchefsky AS. Clinical occult breast lesions; Localization and significance. Radiology 1987;162: $167-170$.

Roux C, Coatrieux JL. Contemporary perspectives in three-dimensional biomedical imaging. Amsterdam, The Netherlands: IOS Press, 1997.

Shankar PM, Reid JM, Ortega H, et al. Use of non-Rayleigh statistics for identification of tumors in ultrasonic B-scans of the breast. IEEE Trans Med Imaging 1993;12:687.

Srihari RK. Automatic indexing and content-based retrieval of captioned images. IEEE Comput 1995;28:49-56.

Stavros T, Thickman D, Rapp CL, Dennis MA, Parker SH, Sisney GA. Solid breast nodules: Use of sonography to distinguish between benign and malignant lesions. Radiology 1995;196:123-134.

Stomper PC, Herman S, Klippenstein DL, et al. Suspect breast lesions: Findings at dynamic gadolinium-enhanced MR imaging correlated with mammographic and pathologic features. Radiology 1995;197: 387-395.

Tiling R, Sommer H, Pechmann M, et al. Comparision of technetium$99 \mathrm{~m}$-sestamibi scintimammography with contrast-enhanced MRI for diagnosis of breast lesions. J Nucl Med 1997;38:58-62.

Vittitoe NF, Baker JA, Floyd CE, Jr. Fractal texture analysis in computer-aided diagnosis of solitary pulmonary nodules. Acad Radiol 1997;4:96-101. 